\title{
Positron beam extraction from an electron-beam-driven plasma wakefield accelerator
}

\author{
H. Fujii®, ${ }^{1}$ K. A. Marsh, ${ }^{1}$ W. An, ${ }^{1}$ S. Corde $\odot,{ }^{2}$ M. J. Hogan, ${ }^{3}$ V. Yakimenko, ${ }^{3}$ and C. Joshi ${ }^{1}$ \\ ${ }^{1}$ University of California, Los Angeles, Los Angeles, California 90095, USA \\ ${ }^{2}$ LOA, ENSTA Paris, CNRS, Ecole Polytechnique, Institut Polytechnique de Paris, \\ 91762 Palaiseau, France \\ ${ }^{3}$ SLAC National Accelerator Laboratory, Menlo Park, California 94025, USA
}

(Received 5 July 2019; published 5 September 2019)

\begin{abstract}
The extraction of accelerated positrons from the electron-beam-driven nonlinear plasma wakefield is investigated. If the trailing positron beam traverses a plasma density down-ramp at the exit of the plasma, it can become ring-shaped due to the defocusing force of the elongating wake bubble produced by the electron drive bunch. Ionization-induced head erosion process can be used to mitigate this effect because the drive electron beam leaves a hollow plasma channel with zero focusing force along the propagation axis. Particle-in-cell simulations are performed for the case where a drive-trailing electron bunch configuration traverses through a high- $Z$ tantalum target placed at the plasma entrance, during which each bunch generates electron-positron pairs. If the plasma density upstream of this converter foil is correctly chosen such that the bubble length is equal to the inter-bunch spacing then the secondary positrons experience both a focusing and accelerating force and can gain energy from the wake. The simulation results demonstrate that when the uniform plasma length is longer than the beam head erosion distance for the drive electron bunch, the positrons can be extracted with a far smaller divergence than when they are extracted by the usual plasma density down-ramp. The transverse density distribution and the positron spectra of the trailing positrons for various acceptance angles of the spectrometer are presented to discuss the detectability of the accelerated positrons under experimental conditions at FACET-II.
\end{abstract}

DOI: 10.1103/PhysRevAccelBeams.22.091301

\section{INTRODUCTION}

The plasma wakefield accelerator (PWFA) is a promising advanced accelerator concept for realizing high gradient accelerators. While much progress has been made in PWFA field with electrons, much remains to be done with respect to positrons for a future collider application of this scheme. Both the Final Focus Test Beam (FFTB) and the Facility for Advanced Accelerator Experimental Tests (FACET) have conducted PWFA experiments using a high-quality positron bunch as a driver [1-4].

A new facility FACET-II is now nearing completion at SLAC National Accelerator Laboratory for advanced accelerator research including on plasma wakefield acceleration. This facility in the first instance will not have a positron beam capability. It is important therefore to explore alternate ways of generating positrons for injection into electron beam driven wake. We are therefore planning

Published by the American Physical Society under the terms of the Creative Commons Attribution 4.0 International license. Further distribution of this work must maintain attribution to the author(s) and the published article's title, journal citation, and DOI. to test an idea proposed several years ago by X. Wang et al. $[5,6]$ for a compact positron source. This idea uses the drive-trailing electron bunch pair generated by FACET-II and converts them into electron-positron pairs in a thin but high- $Z$ foil placed at the entrance of the plasma. In this scheme, two electron bunches impinge upon a high- $Z$ foil target to produce secondary $e^{-}-e^{+}$pairs as shown in Figs. 1(a) and 1(b). By controlling the spacing between the two electron bunches that produce the $e^{-}-e^{+}$pairs and the plasma density, the pairs produced by the trailing electron bunch can be placed at the exact phase, where plasma electrons expelled by the first drive electron bunch converge back on the axis at the back of the wake [Fig. 1(c)]. The plasma density in this region can far exceed the ion density producing a focusing force on the injected positrons but a defocusing force on the injected electrons.

Electron beam ionized column of lithium (Li) vapor has been employed as a plasma source in PWFA experiments for over 15 years [7,8]. In this source, the $\mathrm{Li}$ vapor is confined by helium (He) gas at either end in a heat-pipe oven. The transition from pure $\mathrm{Li}$ to pure $\mathrm{He}$ in the boundary regions is typically $10 \mathrm{~cm}$ long. It forms a density up-ramp at the entrance and a density down-ramp at the exit of the uniform Li plasma region if the heat pipe is 


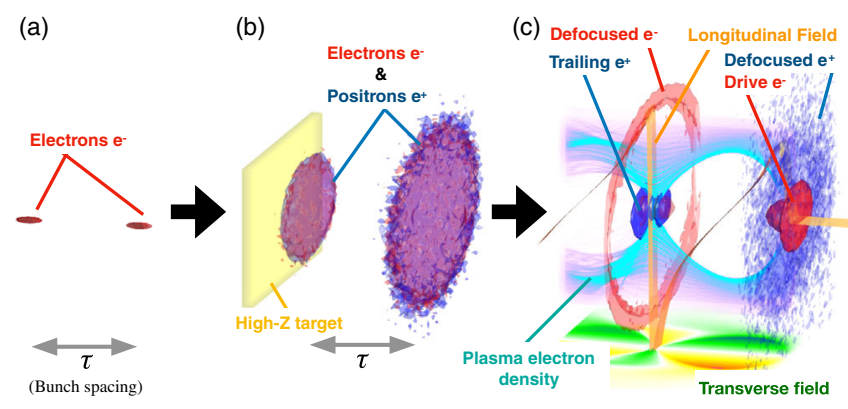

FIG. 1. Schematic diagram of the positron acceleration.

open-ended. In the proposed experiment, the positrons are produced by passing the electron bunches through a high- $Z$ foil which blocks the upstream plasma flow. Thus, there will be no density ramp at the plasma entrance. At the plasma exit, however, the wake wavelength increases as the drive electron beam traverses the down-ramp. Since both the drive bunch and the accelerating positron bunch propagate at the speed of light, the relative spacing between them remains constant and the positrons enter the ion-rich body of the elongating wake and experience a defocusing transverse electric field. This transverse field strongly defocuses the positron beam into a ring as the accelerated positrons pass through the region where down-ramp exists. The positron beam exiting the plasma will therefore not be high quality. One may try to avoid this issue by terminating the plasma with a thin foil at the exit of the uniform region of the plasma. However, further emittance growth by Coulomb scattering is inevitable in this method.

In this paper, we first illustrate this defocusing issue of positrons in the down-ramp region using the particle-in-cell (PIC) simulations. Then, we explore an alternate method for extracting positrons that may reduce the beam degradation at the exit of the plasma. This method uses a lithium gas column longer than the head erosion length of the drive beam. The simulations using typical parameters for the two electron bunch configuration that will be available at FACET-II show that it will be possible to do a systematic exploration of the feasibility of this method of positron generation and acceleration in wakes produced by a drive electron beam at FACET-II.

\section{DOWN-RAMP EXTRACTION}

\section{A. Typical simulation results}

Figure 2 illustrates the positron defocusing issue for down-ramp extraction. Figure 2(a) shows the Li density profile whose 10\%-90\% Gaussian shaped down-ramp length is around $10 \mathrm{~cm}$. In the flat-top density region of the plasma that follows the tantalum (Ta) foil, the beam envelope of the drive beam oscillates around the matched

(a)

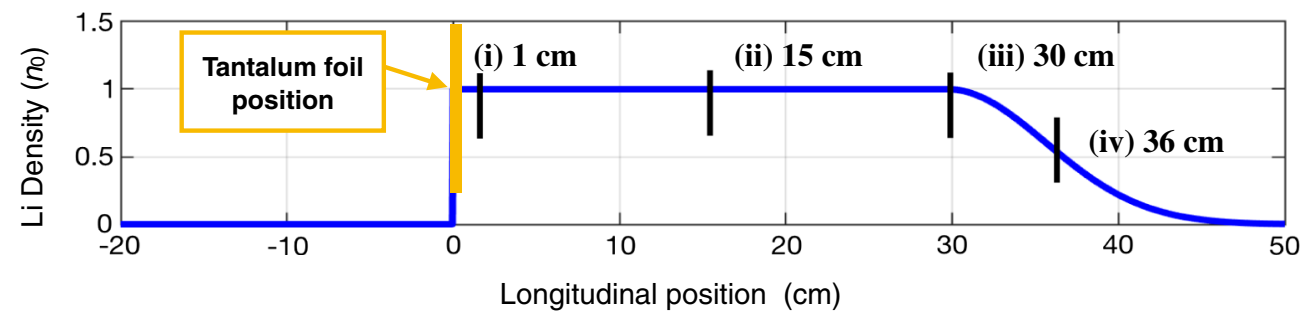

(b)
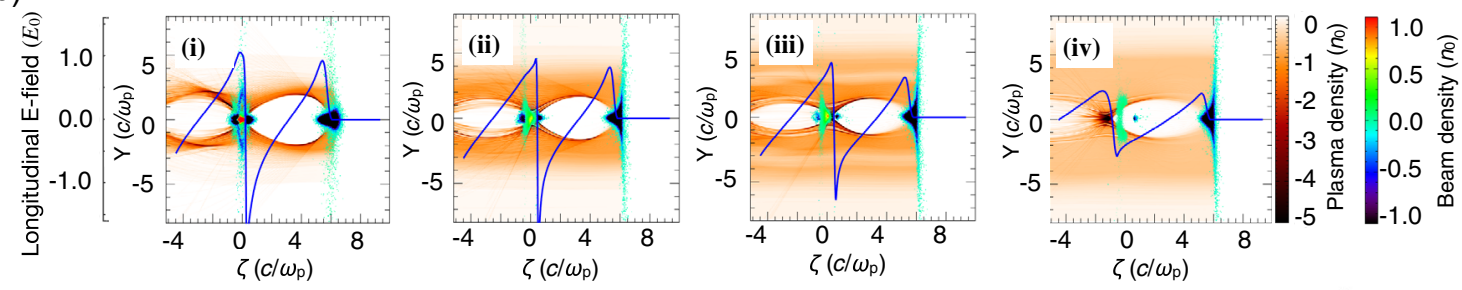

(c)
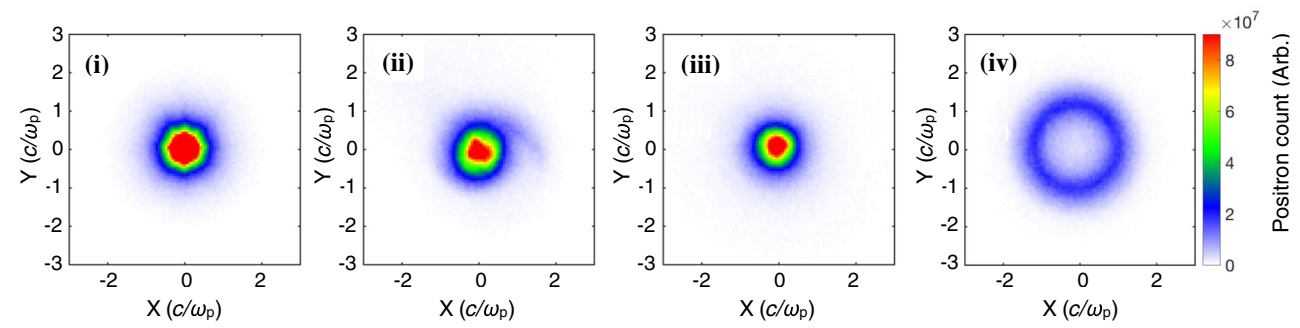

FIG. 2. (a) A typical profile of the plasma and the down-ramp following the tantalum foil. The longitudinal position of the tantalum target is shown by a yellow bar. Black bars (i)-(iv) indicate where figures (b) and (c) are evaluated. (b) Time-evolution of the plasma electron density for the field ionized plasma (red color bar), the beam density (rainbow color bar), and the longitudinal electric field along the central axis. (c) Transverse spatial distribution of accelerated positrons at each position in figure (a). 
beam radius [9]. The tantalum target is placed at $z=0 \mathrm{~cm}$ in all the simulations.

The results shown in Figs. 2(b) and 2(c) are computed first by calculating the interaction of the drive-trailing electron beams with the target using the Monte Carlo simulation code Geant4 [10-12]. Then the 6D phase space distribution of both the primary electron beams and the secondary electron and positron beams is directly imported into the 3D parallel quasistatic particle-in-cell code QuickPIC $[13,14]$. The moving window size is $400 \mu \mathrm{m} \times 400 \mu \mathrm{m} \times$ $380 \mu \mathrm{m}$, and each dimension is divided into 512 grids. The simulation beam parameters before the target are chosen to be similar to the FACET-II design parameters [15]. For simplicity, beam profiles of both the drive and trailing beams are assumed to be identical and Gaussian. Other parameters are as follows: the normalized emittance $\epsilon_{N}=20 \mathrm{~mm}-\mathrm{mrad}$, beam length $\sigma_{z}=6.5 \mu \mathrm{m}$, matched beam radius $\sigma_{r} \equiv \sqrt{\epsilon_{N} / \gamma_{0} k_{\beta}} \sim 2.2 \mu \mathrm{m}$, where $k_{\beta}$ is the betatron wave number given by $k_{\beta}=\omega_{p} /\left(c \sqrt{2 \gamma_{0}}\right), n_{0}=$ $4.5 \times 10^{16} \mathrm{~cm}^{-3}$ is the plasma density, $\omega_{p}$ is the plasma frequency, $c$ is the speed of light and $\gamma_{0}$ is the Lorentz factor of the $10 \mathrm{GeV}$ beam. The charge delivered to the target totals $Q_{d}=1.0 \mathrm{nC}$ for the driver and $Q_{t}=0.5 \mathrm{nC}$ for the trailing bunch, the beam centers are spaced by $150 \mu \mathrm{m}$, where the trailing beam is located at $\zeta \equiv z-c t=0$ and moving in $+z$ direction. The interaction of these beams with the target reduces their average energy to $10 \mathrm{GeV} \times$ $\exp \left(-\Delta z / X_{0}\right)=7.8 \mathrm{GeV}$ as they pass through the $\Delta z=$ $1 \mathrm{~mm}\left(\sim 0.25 X_{0}\right)$ Ta target, where the radiation length $X_{0}$ is $4.094 \mathrm{~mm}$ for Ta. The neutral background lithium gas in the simulation is ionized by the drive beam's self-field according to the Ammosov-Delone-Krainov (ADK) model [16]. The transverse electric field of the beam is intense enough to ionize an $80 \mathrm{~cm}$ long Li column until the front of drive beam completely erodes by diffraction or by giving up its energy to the wake (pump depletion) as long as the $\mathrm{Li}$ column is longer than this length.

Figure 2(b) shows typical simulation results for a $30 \mathrm{~cm}$ long uniform density plasma with a $10 \mathrm{~cm}$ long density ramp on the exit end. Plasma density profiles (red gradation), beam density profiles (rainbow gradation) and longitudinal electric fields along the beam axis (blue solid line) at four different locations (i-iv) shown in Fig. 2(a). Just after the interaction with the target at the point (i), the trailing electrons, shown by the blue gradation color at the intersection of plasma electrons on the beam axis, are being expelled. Throughout the region (ii) to (iii), the wake shape does not evolve and this is where the trailing positrons are accelerated. Then, as the drive bunch enters the plasma down-ramp region the wake elongates adiabatically throughout the region (iv) and the trailing positrons now enter a decelerating and defocusing region of the bubble.

Figure 2(c) shows the transverse density distribution of the trailing positron bunch. The positron beam is transported without being dispersed in the flat-top region (i)-(iii).
At the down-ramp position (iv), the trailing positron forms a ring as the wake elongates as shown in Fig. 2(b)(iv) and thus the trailing positron beam now experiences a defocusing force due to the ions within the plasma wake. This defocusing force reaches values on the order of the normalized electric field $E_{0} \equiv m c \omega_{p} / e \sim 20 \mathrm{GeV} / \mathrm{m}$, which makes it very challenging to bring them back to the central axis using standard magnetic optics.

\section{B. Divergence angle dependence on down-ramp length}

Figure 3 shows the variation of root-mean-square (r.m.s.) angle dependence on the down-ramp length and energy of the accelerated positrons. The transverse cross-sections of the accelerated positron bunch are shown in Fig. 3 for different Gaussian down-ramp lengths $L$ defined as the distance where the density drops from $90 \%$ to $10 \%$ of the peak density after a $30 \mathrm{~cm}$ long region of the uniform plasma. The particles for the r.m.s. angle plots are sampled in the range from $-\sigma_{z}$ to $\sigma_{z}$ relative to the center of the trailing positron bunch after they passed through the $10 \%$ of the peak plasma density. As expected the low energy positrons have a larger divergence angle as the beam divergence typically scales as $1 / \gamma$ for a given defocusing field.

The r.m.s. angle is the largest for the centimeter scale down-ramp length. Thereafter it gradually decreases as the ramp length gets longer. For the case (d) $L=10 \mathrm{~cm}$, we can observe that the r.m.s. angle is similar to the no downramp case. This is because when $L$ is large as in the case (d), the longitudinal focusing phase does not shift

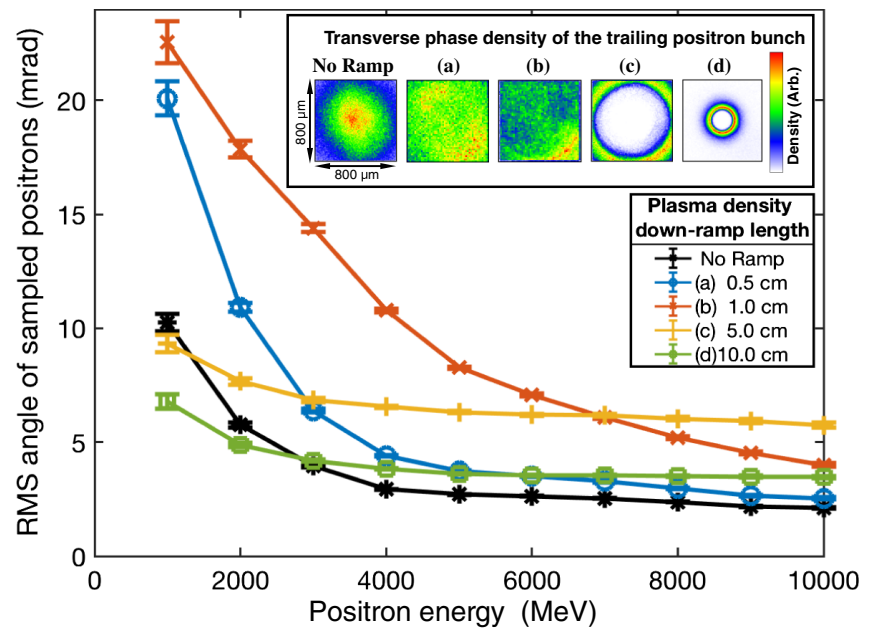

FIG. 3. The divergence angle of positrons as a function of energy $E$ for different down-ramp scale lengths. Each bin is evaluated for positrons in the energy range from $E-\Delta E$ to $E+\Delta E$ where $\Delta E / E=0.05$. These divergence plots are shown after $10 \%$ of the peak plasma density. The particles are sampled in the range $-\sigma_{z}$ to $\sigma_{z}$ of the center of the trailing positron bunch. The corresponding transverse beam profiles at $z=41 \mathrm{~cm}$ are shown on the top-right window. 
significantly along the axis. As a result, positrons form a smaller ring as $L$ is increased.

\section{HEAD EROSION EXTRACTION}

\section{A. Head erosion after the high- $Z$ target}

Energy depletion and head erosion are the two limiting factors that determine the maximum propagation distance of the drive beam inside the beam-ionized plasma [17]. The head erosion occurs because electrons within and ahead of the ionization front are not guided by the ion channel. Therefore, the portion of the beam ahead of the ionization front expands as if propagating in vacuum. Second, once there is an onset of ionization, the ionization front itself will have a certain thickness where the focusing force is still increasing because $\mathrm{Li}$ atoms are only partially ionized [18]. Here the drive beam particles are only partially guided. This leads to the ionization front to gradually recede backward in the beam frame. Once the head erosion process has progressed to a point where the drive bunch cannot ionize the $\mathrm{Li}$, the positron bunch propagates as though in a vacuum: hereinafter referred to as head erosion extraction.

In the beam-driven PWFA, the head erosion of the drive beam can lead to the "wakeless" regime [19]. The transverse electric field at a radius $R$ of the Gaussian beam with peak current $I_{p}$ takes the following form [20]

$$
E_{r}(R)=-\frac{2 I_{p}}{c R} e^{-\tau^{2} / 2 \sigma_{z}^{2}}\left(1-e^{-R^{2} / 2 \sigma_{r}^{2}}\right)
$$

where $\tau=0$ is the beam center along $z$. As the drive beam head erodes, the beam size $\sigma_{r}$ of the ionization front becomes larger, and only a fraction of the radial electric field above the ionization threshold contributes to the tunnel ionization. Below a certain ion column thickness, the plasma electrons cannot gain enough transverse energy to go back to the central axis at the plasma wavelength as in the blowout regime. Therefore, the passage of the drive electron beam leaves a neutral region along the central axis. The accelerated positrons can be extracted through this hollow plasma channel without forming a ring.

The head erosion or etching rate $V(\mu \mathrm{m} / \mathrm{m})$ is defined as the recession distance per relative position of the ionization contour of the drive beam. In Ref. [21], the beam etching rate for a flattop beam is found to scale as

$$
V \propto \frac{\epsilon_{N}}{\gamma} \frac{1}{I^{3 / 2}}
$$

where $I$ is the beam current, $\epsilon_{N}$ is the normalized emittance, and $\gamma$ is the Lorentz factor of the drive beam. For the positron acceleration experiment at FACET-II, the parameters are variables of a high- $Z$ target thickness. When the drive beam passes through the target, the normalized emittance $\epsilon_{N}$ degrades, the primary electron current $I$ reduces because of the phase slippage of low energy electrons after generating $e^{-}-e^{+}$pairs. Also, the mean energy of primary electrons depletes by bremsstrahlung radiation as a function of $\gamma=\gamma_{0} \exp \left(-\Delta z / X_{0}\right)$ which defines the radiation length. The scaling law Eq. (2) tells that the emittance growth, the current reduction and the reduction of $\gamma$ by the interaction with the target lead to a higher rate of the head erosion.

The ionization front for the Gaussian beam is determined by the maximum field strength of the drive beam. According to Blumenfeld [20], the head erosion rate varies in time for the Gaussian beam. The ionization front position relative to the beam center $s_{\text {ion }}$ is at

$$
\begin{aligned}
s_{\text {ion }}= & \frac{2 \sigma_{z}[\mu \mathrm{m}]}{\sqrt{3}} \\
& \times \operatorname{erf}^{-1}\left(\operatorname{erf}(\sqrt{3})-\sqrt{\frac{3}{\pi}} \frac{1}{\sigma_{z}[\mu \mathrm{m}]} \frac{\alpha}{I_{p}^{3 / 2}[\mathrm{kA}]} z[\mathrm{~m}]\right)
\end{aligned}
$$

where we plugged in the peak current of drive beam $I_{p}=Q_{d} c / \sqrt{2 \pi} \sigma_{z} \sim 18.4 \mathrm{kA}$ and

$$
\alpha=\left(3.6617 \times 10^{4}\right) E_{i}^{1.73} \frac{\epsilon_{N}}{\gamma},
$$

where $E_{i}=5.39 \mathrm{eV}$ is the ionization energy for lithium gas.

The divergence angle after the multiple Coulomb scattering is derived using Highland's formula [22]

$$
\theta=13.6 \frac{\sqrt{\Delta z / X_{0}}}{p \beta}\left[1+0.088 \log _{10}\left(\Delta z / X_{0}\right)\right]
$$

as a variable of the normalized target thickness $\Delta z / X_{0}$, where $p$ is the momentum of incident electrons. Due to this appreciable change in angle, the emittance $\epsilon$ after the multiple Coulomb scattering is given by [23]

$$
\epsilon=\left(\epsilon_{0}^{2}+\beta_{0} \epsilon_{0} \theta\right)^{1 / 2},
$$

where $\beta_{0} \equiv 1 / k_{\beta}$ is the Twiss parameter at the beam waist inside the plasma, $\epsilon_{0}=\epsilon_{N 0} / \gamma_{0}$ is the initial geometric emittance.

The range of $z$ over which the inverse error function of $s_{\text {ion }}$ is finite in Eq. (3) tells the maximum distance the drive beam can propagate. This theoretical limit is plotted in Fig. 4 by taking the reduction of the mean energy and the emittance growth predicted by Highland into account. The beam parameters in the Sec. II A are used except that the initial normalized emittance $\epsilon_{N 0}$ is varied for both drive and trailing beams, and thus the beam radii $\sigma_{r}$ are changed according to the matched beam radius for the plasma density $n_{0}=4.5 \times 10^{16} \mathrm{~cm}^{-3}$. In the same figure, the ionization distances simulated for different target thickness $\Delta z=0.5,1$ and $2 \mathrm{~mm}$ are shown. The results are shown 


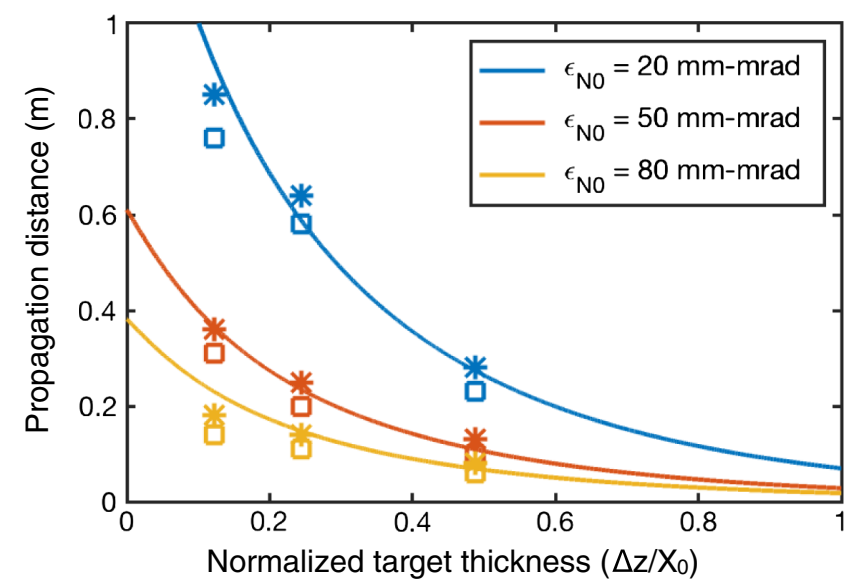

FIG. 4. The maximum head erosion distance dependence predicted by Eq. (3) are shown by solid lines for different initial emittance values $\epsilon_{N 0}$. The square symbols indicate the distances where the density begins to drop down and the asterisk symbols are when the density reaches zero at the tail of the drive electron beam $\left(-3 \sigma_{z}\right.$ relative to the drive beam center) in PIC simulations.

for the distance when the plasma density starts to decrease (square symbols) and becomes zero (asterisk symbols) at the tail of the drive beam $\left(-3 \sigma_{z}\right)$. The similar results of the theoretical curve and the simulations seen in Fig. 4 shows the validity of the scaling discussed from Eq. (3) to Eq. (6).

\section{B. Phase space distribution and the positron spectrum}

We evaluate the quality of the head erosion extraction for the $\Delta z=2 \mathrm{~mm}$ Ta thickness and $\epsilon_{N 0}=20 \mathrm{~mm}-\mathrm{mrad}$ initial emittance case. As was shown in Fig. 4, the plasma density starts to decrease at $23 \mathrm{~cm}$ and becomes zero at
$28 \mathrm{~cm}$ at the tail of the drive beam. In the following simulation results, the plasma is infinitely long and has a constant density in the head erosion extraction case. For the density down-ramp extraction case, the plasma has a density down-ramp $L=10 \mathrm{~cm}$ after $20 \mathrm{~cm}$ uniform region [See Fig. 5(a)], i.e., the ramp begins just before the wake begins to show degradation due to head erosion.

Figure 5(b) shows the plasma electron density in Y-Z plane in the head erosion extraction case for each indicated position in Fig. 5(a). Figure 5(c) is the lineout of the transverse field $E_{r}-B_{\theta}$ experienced by the trailing positron bunch center $\zeta=0$. The effect of the head erosion manifests as the formation of a hollow plasma channel with increasing diameter. The reduction of the plasma density on the axis around the trailing beam in $31 \mathrm{~cm}$ case leads to the similar reduction and eventual disappearance of the focusing force allowing the positron bunch to propagate through the rest of the Li vapor without significant defocusing.

In the density plots Figs. 6 and 7, the group (a) shows the head erosion extraction case whereas the group (b) shows the down-ramp extraction case. Both cases are generated by sampling particles at $z=31 \mathrm{~cm}$ at which point the accelerated particles are still within the QuickPIC simulation window. Particles used for the density plots in Figs. 6 and 7 are sampled after numerically masking the particles using a collimator. In the following discussion, $5 \mathrm{mrad}$ or $2 \mathrm{mrad}$ acceptance refers to the filtering of the particles by putting a $5 \mathrm{~mm}$ or $2 \mathrm{~mm}$ radius apertures in the detection plane at $z=131 \mathrm{~cm}$. In addition to that, the transverse spatial density and the phase density are sampled for the particles in the region within $-\sigma_{z}$ to $\sigma_{z}$ relative to the beam center.

Figures 6(a1) and 6(b1) show the transverse spatial density of each case. Some particles reside in the center

(a)

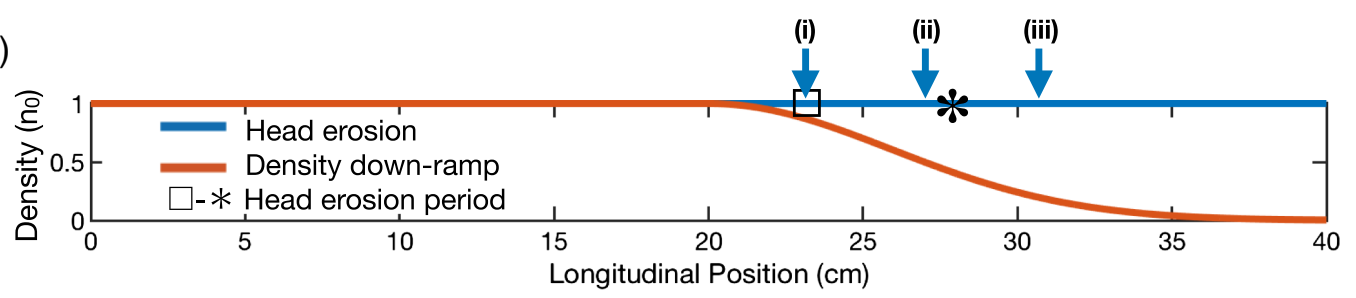

(b)

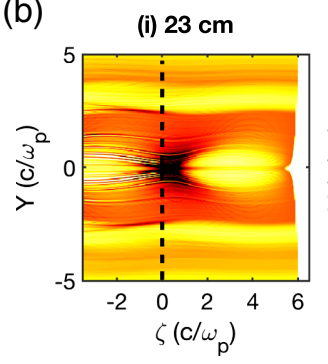

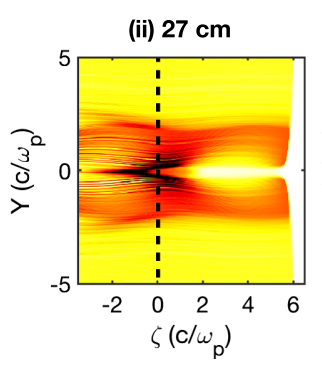

(c)



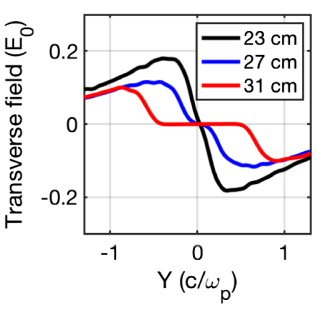

FIG. 5. (a) The density profile used for the comparison of head erosion extraction (blue) and down-ramp extraction (red). Square and asterisk symbols indicate the beginning and the completion of the head erosion process for the uniform plasma case as in Fig. 4. (b) Plasma electron density for the head erosion extraction case at three different positions (i), (ii), and (iii) in the figure (a). (c) Lineouts of the transverse field at the dashed line in the figure (b). The flatness of the field indicates that there is no focusing force on the positrons. 


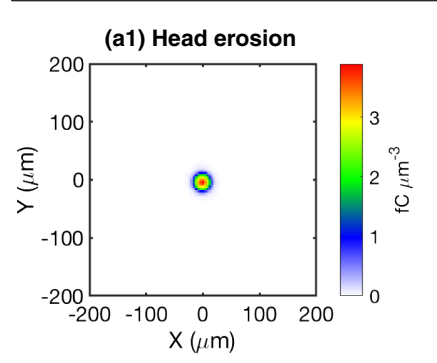

(a2) Head erosion
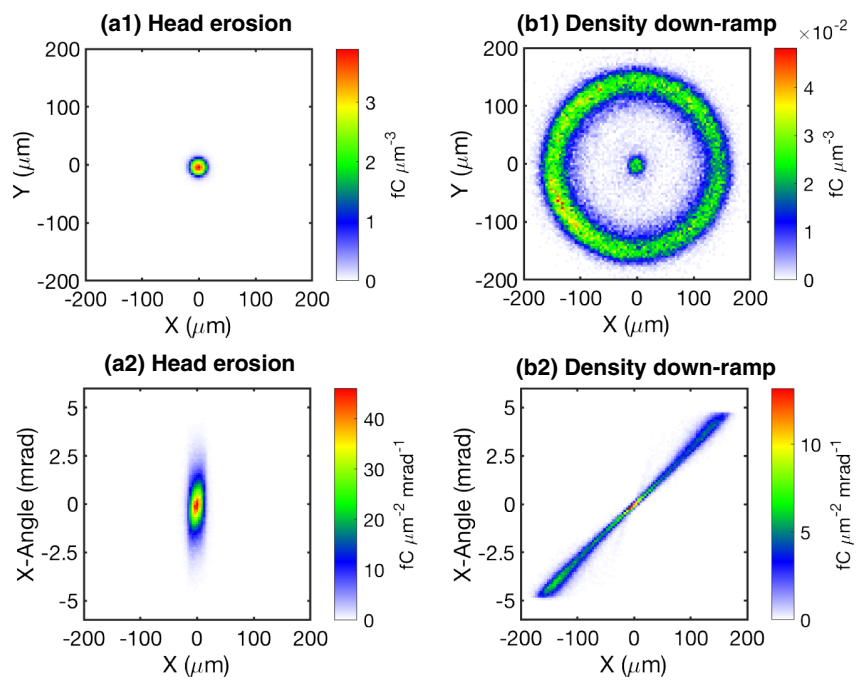

FIG. 6. The density distributions of trailing positrons after the 5 mrad acceptance transport system for (a) head erosion extraction and (b) $10 \mathrm{~cm}$ down-ramp extraction. The top figures (a1) and (b1) shows the transverse spatial density, the bottom figures (a2) and (b2) shows the phase space density of particles in the range. The particles are sampled in the range $-\sigma_{z}$ to $\sigma_{z}$ of the center of the trailing positron bunch.

of Fig. 6(b1) while these are not observed in the right-top windows in Fig. 3, the latter being due to the combined effect of the down-ramp and head erosion. Figure 6(a1) indicates the head erosion process does not cause a large angular dispersion of the positron beam. Figures 6(a2) and 6(b2) show the transverse phase space density of each group. The color scale of the group (a) shows that the larger number of particles still remains near the axis in the head erosion extraction case, while the particles are strongly defocused in the down-ramp extraction case (b).

Figure 7 shows the same physical quantities as in Fig. 6 except that sampling particles are filtered by a $2 \mathrm{mrad}$ transport system. With this acceptance, we no longer see the characteristic ring which is blown out from the axis by the ion channel when a down-ramp exists, whereas both the transverse profile and the phase space plot are almost identical when we rely on the head erosion.

Figure 8(a) shows the energy spectra of positrons at $z=0 \mathrm{~cm}$ (black) and after head erosion extraction (blue) and down-ramp extraction (red) at $z=31 \mathrm{~cm}$. The peaks of the red and the blue solid lines indicate that the positrons gained around $2 \mathrm{GeV}$ in $20 \mathrm{~cm}$. Figure 8(b) shows the spectra when the beam transport system that follows the plasma oven has either a $5 \mathrm{mrad}$ or $2 \mathrm{mrad}$ acceptance angle. The black curve (5 mm aperture) in Fig. 8(b) is the positron spectrum at the detection plane without the plasma. In a previous conference publication [24], we have shown the divergence angle of the positrons as a function of the energy at the exit of the high- $Z$ foil. As expected, the lower energy positrons have a higher divergence angle $\theta$ and that it decreases as the positron energy increases since
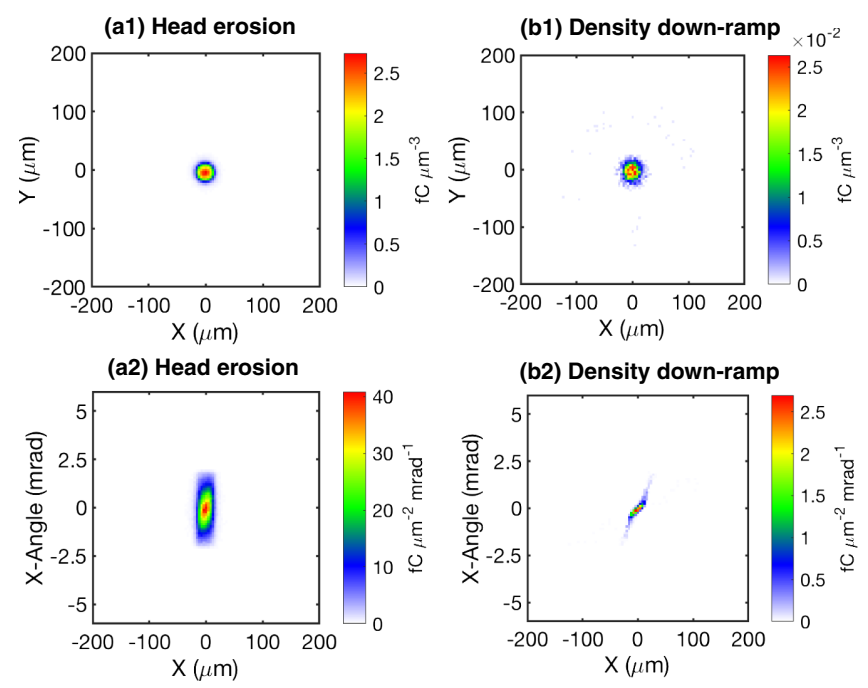

FIG. 7. Counterpart of Fig. 6 with the $2 \mathrm{mrad}$ acceptance transport system.
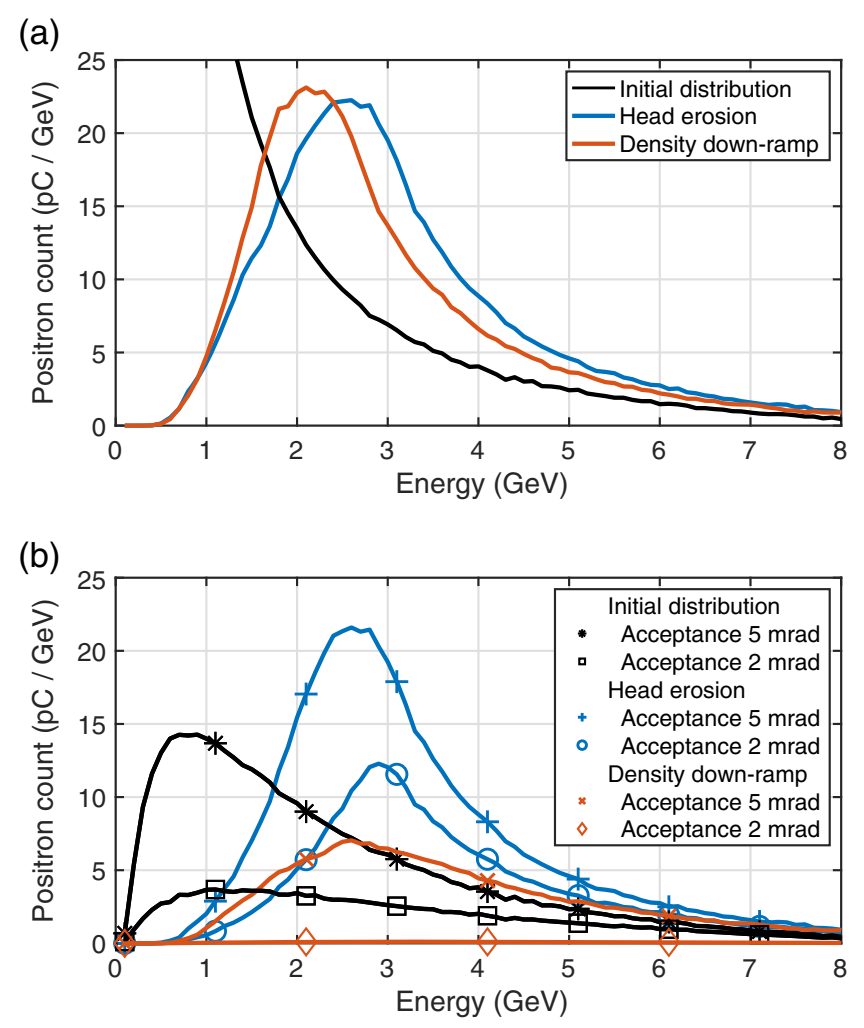

FIG. 8. (a) The initial energy distribution just after the target (black), the accelerated energy distribution in the head erosion extraction (blue) case and the down-ramp extraction (red) case evaluated at the distance of $z=31 \mathrm{~cm}$. (b) A counterpart of the figure (a) after passing through the $5 \mathrm{mrad}$ and $2 \mathrm{mrad}$ acceptance angle systems respectively. 
$\theta=p_{\perp} / p_{z} \propto 1 / \gamma$. The blue curve (5 mm aperture) is after the capture and the acceleration of most of these positrons by the plasma wake. The plasma focusing force captures positrons and the energy gain in the longitudinal direction reduces the divergence angle of the accelerated particles and therefore increases the number of positrons at the detection plane. Therefore the transmitted fractions of the beam through both the $2 \mathrm{~mm}$ and $5 \mathrm{~mm}$ apertures are greater in the beam head erosion case than in the respective case for the initial distribution without the plasma.

For the $5 \mathrm{mrad}$ acceptance case, almost all the positrons are transported in the head erosion case while in the downramp extraction case only less than half the accelerated positrons are transported. For the 2 mrad acceptance, few positrons are transported for the down-ramp extraction case. The spectra shown in Fig. 8(b) and the density plots shown in Fig. 7 both indicate that head erosion extraction is advantageous over down-ramp extraction. The peak of the positron spectrum in the head erosion extraction case has between $10-20 \mathrm{pC} / \mathrm{GeV}$ charge which should be detectable in an experiment.

\section{CONCLUSION}

This work demonstrates the wakefield acceleration of positrons in plasmas formed by the Coulomb field of a drive electron bunch. The plasma down-ramp defocusing issue in positron extraction is solved by propagating the drive beam until it completely head erodes. The injected positrons are accelerated at an average gradient of $5-10 \mathrm{GeV} / \mathrm{m}$. The accelerated spectrum after a collimator shows several $\mathrm{pC} / \mathrm{GeV}$ positron signal that should be detectable for the parameters of the trailing electron bunch and the target used to generate the positron bunch.

We have investigated positron extraction from a nonlinear wakefield structure. However, linear wakes may well have a better charge capture efficiency for the trailing bunch [24,25]. Linear wakefields can be excited by changing the ratio of the original two electron beams and the high- $Z$ target thickness. The efficiency of capturing and extraction of positrons from a beam-ionized linear plasma wakefield needs to be further investigated.

In this paper, positrons are generated by using a drivetrailing electron configuration planned at FACET-II. However, the same positron extraction issues arise when extracting an externally injected positron bunch in an electron beam produced wake.

\section{ACKNOWLEDGMENTS}

This work was performed under the auspices of the U.S. DOE Grant No. DE-SC0010064 and the U.S. NSF Grant No. PHY-1734315. Simulations are performed on Hoffman cluster at UCLA and Edison cluster at National Energy Research Scientific Computing Centre (NERSC).
[1] B. E. Blue, C. E. Clayton, C. L. O'Connell, F.-J. Decker, M. J. Hogan, C. Huang, R. Iverson, C. Joshi, T. C. Katsouleas, W. Lu et al., Plasma-Wakefield Acceleration of an Intense Positron Beam, Phys. Rev. Lett. 90, 214801 (2003).

[2] S. Corde, E. Adli, J. M. Allen, W. An, C. I. Clarke, C. E. Clayton, J. P. Delahaye, J. Frederico, S. Gessner, S. Z. Green et al., Multi-gigaelectronvolt acceleration of positrons in a self-loaded plasma wakefield, Nature (London) 524, 442 (2015).

[3] A. Doche, C. Beekman, S. Corde, J. M. Allen, C. I. Clarke, J. Frederico, S. J. Gessner, S. Z. Green, M. J. Hogan, B. O'Shea et al., Acceleration of a trailing positron bunch in a plasma wakefield accelerator, Sci. Rep. 7, 14180 (2017).

[4] S. Gessner, E. Adli, J. M. Allen, W. An, C. I. Clarke, C. E. Clayton, S. Corde, J. P. Delahaye, J. Frederico, S. Z. Green et al., Demonstration of a positron beam-driven hollow channel plasma wakefield accelerator, Nat. Commun. 7, 11785 (2016).

[5] X. Wang, R. Ischebeck, P. Muggli, T. Katsouleas, C. Joshi, W. B. Mori, and M. J. Hogan, Positron Injection and Acceleration on the Wake Driven by an Electron Beam in a Foil-and-Gas Plasma, Phys. Rev. Lett. 101, 124801 (2008).

[6] X. Wang, P. Muggli, T. Katsouleas, C. Joshi, W. B. Mori, R. Ischebeck, and M. J. Hogan, Optimization of positron trapping and acceleration in an electron-beam-driven plasma wakefield accelerator, Phys. Rev. Accel. Beams 12, 051303 (2009).

[7] P. Muggli, K. Marsh, S. Wang, C. Clayton, S. Lee, T. Katsouleas, and C. Joshi, Photo-ionized lithium source for plasma accelerator applications, IEEE Trans. Plasma Sci. 27, 791 (1999).

[8] N. Vafaei-Najafabadi, J. L. Shaw, K. A. Marsh, C. Joshi, and M. J. Hogan, Meter scale plasma source for plasma wakefield experiments, AIP Conf. Proc. 1507, 650 (2013).

[9] K. A. Marsh, W. An, C. E. Clayton, C. Joshi, W. B. Mori, N. Vafaei-najafabadi, P. Muggli, C. I. Clarke, S. Corde, R. J. England et al., in Proceedings of the 25th Particle Accelerator Conference, PAC-2013, Pasadena, CA, 2013 (IEEE, New York, 2013), pp. 1105-1107.

[10] S. Agostinelli, J. Allison, K. Amako, J. Apostolakis, H. Araujo, P. Arce, M. Asai, D. Axen, S. Banerjee, G. Barrand et al., Geant4-a simulation toolkit, Nucl. Instrum. Methods Phys. Res., Sect. A 506, 250 (2003).

[11] J. Allison, K. Amako, J. Apostolakis, H. Araujo, P. Arce Dubois, M. Asai, G. Barrand, R. Capra, S. Chauvie, R. Chytracek et al., Geant4 developments and applications, IEEE Trans. Nucl. Sci. 53, 270 (2006).

[12] J. Allison, K. Amako, J. Apostolakis, P. Arce, M. Asai, T. Aso, E. Bagli, A. Bagulya, S. Banerjee, G. Barrand et al., Recent developments in Geant4, Nucl. Instrum. Methods Phys. Res., Sect. A 835, 186 (2016).

[13] C. Huang, V. Decyk, C. Ren, M. Zhou, W. Lu, W. Mori, J. Cooley, T. Antonsen, and T. Katsouleas, QUICKPIC: A highly efficient particle-in-cell code for modeling wakefield acceleration in plasmas, J. Comput. Phys. 217, 658 (2006).

[14] W. An, V. K. Decyk, W. B. Mori, and T. M. Antonsen, An improved iteration loop for the three dimensional 
quasi-static particle-in-cell algorithm: QuickPIC, J. Comput. Phys. 250, 165 (2013).

[15] C. Joshi, E. Adli, W. An, C. E. Clayton, S. Corde, S. Gessner, M. J. Hogan, M. Litos, W. Lu, K. A. Marsh et al., Plasma wakefield acceleration experiments at FACET II, Plasma Phys. Controlled Fusion 60, 034001 (2018).

[16] M. V. Ammosov, N. B. Delone, and V. P. Krainov, Tunnel ionization of complex atoms and of atomic ions in an alternating electromagnetic field, Sov. Phys. JETP 64, 1191 (1986).

[17] W. An, M. Zhou, N. Vafaei-Najafabadi, K. A. Marsh, C. E. Clayton, C. Joshi, W. B. Mori, W. Lu, E. Adli, S. Corde et al., Strategies for mitigating the ionization-induced beam head erosion problem in an electron-beam-driven plasma wakefield accelerator, Phys. Rev. Accel. Beams 16, 101301 (2013).

[18] I. Blumenfeld, C. E. Clayton, F.-J. Decker, M. J. Hogan, C. Huang, R. Ischebeck, R. Iverson, C. Joshi, T. Katsouleas, N. Kirby et al., Energy doubling of $42 \mathrm{GeV}$ electrons in a metre-scale plasma wakefield accelerator, Nature (London) 445, 741 (2007).

[19] M. Litos, R. Ariniello, C. Doss, K. Hunt-Stone, and J. R. Cary, in 2018 IEEE Advanced Accelerator Concepts Workshop (AAC) (IEEE, New York, 2018), pp. 1-5.

[20] I. Blumenfeld, Ph.D. thesis, Stanford University, 2009.

[21] M. Zhou, Ph.D. thesis, University of California Los Angeles, 2008.

[22] G. R. Lynch and O. I. Dahl, Approximations to multiple Coulomb scattering, Nucl. Instrum. Methods Phys. Res., Sect. B 58, 6 (1991).

[23] J. B. Rosenzweig and P. Chen, Beam optics of a selffocusing plasma lens, Phys. Rev. D 39, 2039 (1989).

[24] H. Fujii, W. An, K. A. Marsh, W. B. Mori, and C. J. Joshi, in 2018 IEEE Advanced Accelerator Concepts Workshop (AAC) (IEEE, New York, 2018), pp. 1-5.

[25] K. V. Lotov, Acceleration of positrons by electron beamdriven wakefields in a plasma, Phys. Plasmas 14, 023101 (2007). 